\title{
Functional Outcomes of Multiple Sural Nerve Grafts for Facial Nerve Defects after Tumor- Ablative Surgery
}

\author{
Myung Chul Lee ${ }^{1}$, Dae Hee Kim ${ }^{1}$, Yeo Reum Jeon ${ }^{1}$, Dong Kyun Rah ${ }^{1}$, Dae Hyun Lew ${ }^{1}$, \\ Eun Chang $\mathrm{Choi}^{2}$, Won Jai Lee ${ }^{1}$ \\ ${ }^{1}$ Department of Plastic and Reconstructive Surgery, Institute for Human Tissue Restoration, ${ }^{2}$ Department of Otorhinolaryngology, \\ Yonsei University College of Medicine, Seoul, Korea
}

Background Functional restoration of the facial expression is necessary after facial nerve resection to treat head and neck tumors. This study was conducted to evaluate the functional outcomes of patients who underwent facial nerve cable grafting immediately after tumor resection.

Methods Patients who underwent cable grafting from April 2007 to August 2011 were reviewed, in which a harvested branch of the sural nerve was grafted onto each facial nerve division. Twelve patients underwent facial nerve cable grafting after radical parotidectomy, total parotidectomy, or schwannoma resection, and the functional facial expression of each patient was evaluated using the Facial Nerve Grading Scale 2.0. The results were analyzed according to patient age, follow-up duration, and the use of postoperative radiation therapy.

Results Among the 12 patients who were evaluated, the mean follow-up duration was 21.8 months, the mean age at the time of surgery was 42.8 years, and the mean facial expression score was 14.6 points, indicating moderate dysfunction. Facial expression scores were not influenced by age at the time of surgery, follow-up duration, or the use of postoperative radiation therapy.

Conclusions The results of this study indicate that facial nerve cable grafting using the sural nerve can restore facial expression. Although patients were provided with appropriate treatment, the survival rate for salivary gland cancer was poor. We conclude that immediate facial nerve reconstruction is a worthwhile procedure that improves quality of life by allowing the recovery of facial expression, even in patients who are older or may require radiation therapy.

Keywords Facial nerve / Sural nerve / Surgery / Radiotherapy / Age groups
Correspondence: Won Jai Lee Department of Plastic and Reconstructive Surgery, Yonsei University College of Medicine, 250 Seongsan-ro, Seodaemun-gu,

Seoul 120-752, Korea

Tel: $+82-2-2228-2215$

Fax: +82-2-393-6947

E-mail: pswjlee@yuhs.ac

No potential conflict of interest relevant to this article was reported.

Received: 28 Feb 2015 - Revised: 22 Apr $2015 \bullet$ Accepted: 8 May 2015

pISSN: 2234-6163 • elSSN: 2234-6171 • http://dx.doi.org/10.5999/aps.2015.42.4.461 • Arch Plast Surg 2015;42:461-468

\section{INTRODUCTION}

Radical parotidectomy involves the total extirpation of the parotid gland along with resection of the facial nerve. Sacrifice of the facial nerve is also unavoidable in patients with facial nerve tumors. In such cases, functional restoration of the facial nerve has proven challenging. Plastic surgeons have introduced many operative techniques, including facial nerve reconstruction with

Copyright (C) 2015 The Korean Society of Plastic and Reconstructive Surgeons

This is an Open Access article distributed under the terms of the Creative Commons Attribution Non-Commercial License (http://creativecommons.org/

licenses/by-nc/3.0/) which permits unrestricted non-commercial use, distribution, and reproduction in any medium, provided the original work is properly cited. I www.e-aps.org 
end-to-end hypoglossofacial anastomosis [1], hypoglossal-facial nerve jump grafting [2], interposition nerve grafting using the great auricular nerve [3], end-to-side nerve grafting for facial nerve reconstruction [4], and an approach combining facial nerve interpositional grafting with hypoglossal-facial jump nerve sutures [5]. These surgical techniques were developed and modified with the aim of achieving natural voluntary facial expressions with symmetrical tone at rest, while minimizing sequelae such as synkinesis and donor site morbidity. Although early nerve reconstruction has been recommended by many authors, few studies have reported outcomes after facial nerve grafting $[6,7]$. The inability to perform facial expressions after ablative operations performed to treat head and neck tumors, such as radical parotidectomy, is both aesthetically and functionally problematic for patients. Therefore, a better understanding of the long-term results of facial nerve grafting is needed.

Facial nerve cable grafting using the sural nerve is a well-established procedure for the repair of facial nerve defects [8]. The sural nerve is one of the most commonly used donor nerves in autologous nerve grafting, because it serves a purely sensory function and can easily be harvested [9]. Moreover, it is a long nerve consisting of multiple fascicles with inter-fascicular bridges. Its morphology and topography, which have been studied by many investigators, allow the creation of three independent nerve grafts from every sural nerve that is dissected [10]. Splitting the sural nerve meticulously into two or three divisions mimics the branching pattern of the original facial nerve [11].

This study was conducted to evaluate functional outcomes in patients who underwent facial nerve cable grafting after ablative surgery was performed to treat head and neck tumors. Facial nerve function was evaluated by plastic surgeons via the Facial Nerve Grading Scale 2.0 (FNGS), based on observations of the patients making facial expressions [12]. The FNGS assesses movements in the brow, eye, nasolabial fold, and oral commissure.

\section{METHODS}

We performed a retrospective review of patients who underwent facial nerve cable grafting with the sural nerve immediately after ablative surgery to treat head and neck tumors at Severance Hospital from April 2007 to August 2011. This study was approved by the Institutional Review Board of the Yonsei University College of Medicine. Of the 16 patients who underwent facial nerve cable graft surgery after radical parotidectomy, total parotidectomy, or schwannoma resection, four patients were excluded because they experienced recurrence of the tumor $(n=2)$, experienced other facial nerve injuries $(n=1)$, or $\operatorname{died}(n=1)$. The fa- cial nerve functionality of the remaining 12 patients was evaluated.

\section{Surgical technique}

Truncated proximal trunk and distal branches were identified after tumor-ablative surgery was performed by otorhinolaryngologists. The distance between the proximal and distal nerve endings was measured, and a curvilinear incision was made between the lateral malleolus and Achilles tendon [9]. The sural nerve was identified subcutaneously, adjacent to the lesser saphenous vein. An adequate amount of the sural nerve was harvested in order to connect the proximal and distal facial nerve stumps. Cutaneous branches were harvested along with the sural nerve trunk in order to imitate the arborescent pattern of facial nerve branches [11]. The harvested nerve was divided into two segments: one for the temporofacial division and one for the cervicofacial division of the facial nerve. The harvested nerve segments were placed in the recipient sites, with the distal portion of the sural nerve on the proximal trunk of the facial nerve and the proximal portion of the sural nerve on the distal branches of the facial nerve, following the direction of neural signaling. The superior and inferior halves of the proximal facial nerve trunk were anastomosed with two segments of the sural nerve stumps in order to achieve cable grafting of two strands of the sural nerve. If additional sural nerve endings were needed for the distal facial nerve branches, the sural nerve was meticulously dissected, preserving the fascicles. Anastomosis of each nerve ending was performed using a perineural technique under microscopic magnification (Fig. 1).

\section{Measurement of facial nerve function}

Facial nerve function was evaluated by plastic surgeons who observed the patients making facial expressions. The evaluation of facial expressions was based on the FNGS 2.0, which is a 2009 revision of the House-Brackmann facial grading system by the Facial Nerve Disorders Committee [12]. In addition, two of the 12 patients were assessed using the FNGS by researchers who reviewed video clips recorded postoperatively in the outpatient department. The FNGS assesses facial movements of the brow, eye, nasolabial fold, and oral commissure, and a score ranging from 1 to 6 is assigned to each region according to the degree of movement. Movement in each facial region was assessed using the same scoring system: normal movement was scored as 1 , slight weakness (75\% of normal movement) was scored as 2 , obvious weakness ( $50 \%-75 \%$ of normal movement) was scored as 3 , asymmetry at rest ( $50 \%$ of normal movement) was scored as 4 , trace movement was scored as 5 , and no movement was scored as 6 . In addition, assessment of synkinesis across the entire face was scored on a scale of 0 to 3 , with maximum points as- 


\section{Fig. 1. Figures illustrating a cable graft procedure}

(A) A facial nerve defect was noted after tumor resection (black and white arrows), and an adequate length of the sural nerve was harvested in order to serve as a cable connecting the proximal and distal facial nerve stumps. Cutaneous branches accompanied the sural nerve trunk, imitating the arborescent pattern of facial nerve branches. (B) The harvested nerve was then divided into two segments, with one corresponding to the temporofacial division of the facial nerve and the other corresponding to the cervicofacial division of the facial nerve. The superior and inferior halves of the proximal facial nerve trunk were anastomosed with two segments of the sural nerve stumps. If additional sural nerve endings were needed for distal facial nerve branches, the nerve was meticulously split, preserving the fascicles. Each nerve ending was anastomosed using a perineural technique under microscopic magnification.
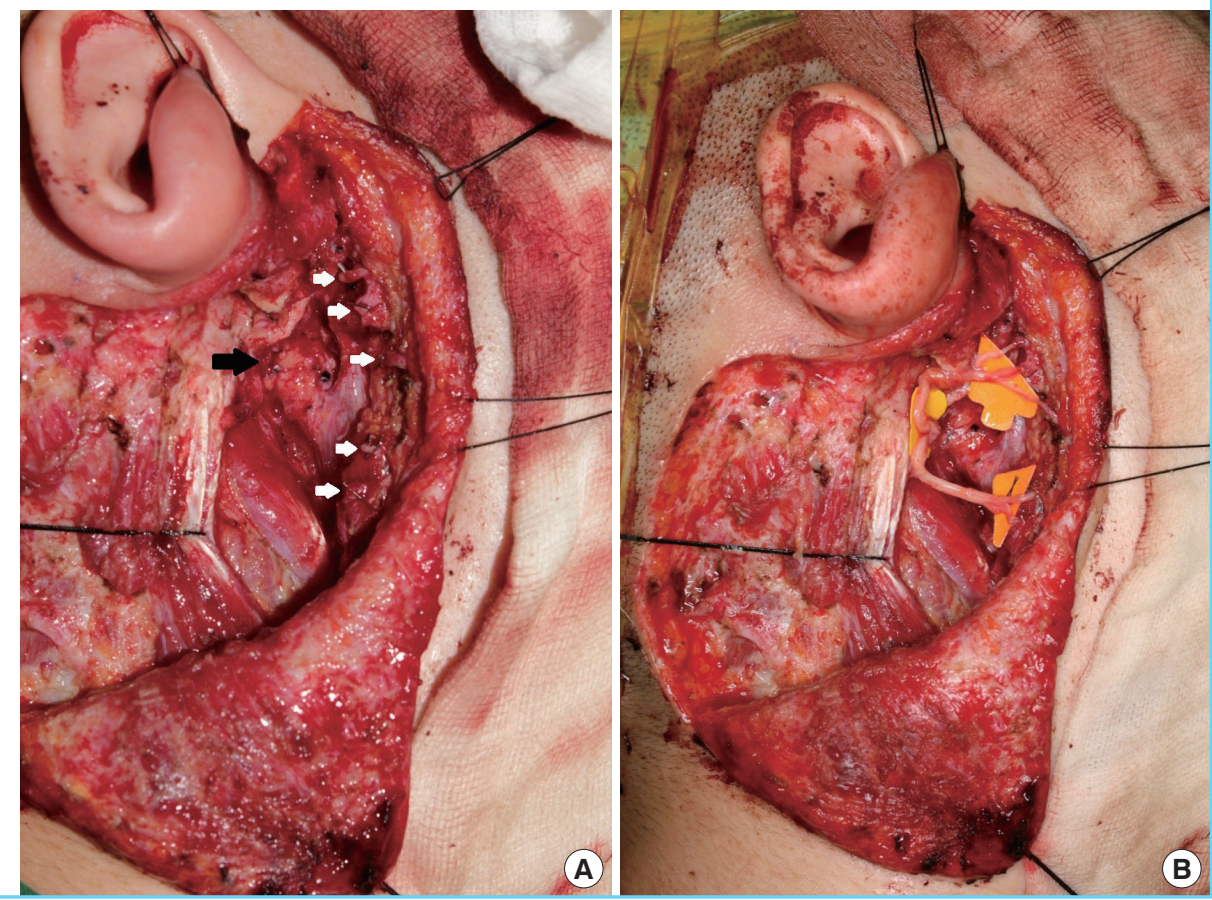

Table 1. Patient demographics

\begin{tabular}{|c|c|c|c|c|c|c|c|c|c|c|c|c|}
\hline Patient & $\begin{array}{l}\text { Age }(y r) / \\
\text { sex }\end{array}$ & $\begin{array}{l}\text { Follow-up } \\
\text { duration } \\
\text { (mo) }\end{array}$ & Diagnosis & Stage & $\begin{array}{c}\text { Radiation } \\
\text { therapy }\end{array}$ & Brow & Eye & $\begin{array}{l}\text { Nasolabial } \\
\text { fold }\end{array}$ & Oral & Synkinesis & $\begin{array}{l}\text { Total } \\
\text { score }\end{array}$ & Grade \\
\hline 1 & 13/Male & 17 & Mucoepidermoid carcinoma & $\|$ & No & 4 & 2 & 1 & 1 & 0 & 8 & $\|$ \\
\hline 2 & 23/Female & 9 & Mucoepidermoid carcinoma & $\|$ & Yes & 5 & 2 & 2 & 2 & 1 & 12 & III \\
\hline 3 & 37/Male & 26 & Mucoepidermoid carcinoma & IVa & Yes & 5 & 3 & 2 & 2 & 0 & 12 & III \\
\hline 4 & 47/Female & 21 & Mucoepidermoid carcinoma & IVa & Yes & 4 & 2 & 3 & 4 & 1 & 14 & III \\
\hline 5 & 64/Male & 61 & Squamous cell carcinoma & $\|$ & Yes & 6 & 4 & 2 & 2 & 0 & 14 & III \\
\hline 6 & 33/Male & 8 & Squamous cell carcinoma & IVa & Yes & 6 & 3 & 3 & 3 & 0 & 15 & IV \\
\hline 7 & 54/Female & 20 & Adenocarcinoma & III & No & 4 & 4 & 3 & 3 & 1 & 15 & IV \\
\hline 8 & 33/Female & 18 & Schwannoma & - & No & 5 & 3 & 3 & 3 & 2 & 16 & IV \\
\hline 9 & 55/Female & 15 & Squamous cell carcinoma & IVa & Yes & 6 & 3 & 3 & 3 & 1 & 16 & IV \\
\hline 10 & 49/Female & 19 & Carcinosarcoma & - & No & 5 & 4 & 3 & 4 & 0 & 16 & IV \\
\hline 11 & 53/Male & 16 & Adenocarcinoma & IVa & Yes & 6 & 5 & 3 & 3 & 0 & 17 & IV \\
\hline 12 & 53/Female & 31 & Schwannoma & - & No & 5 & 5 & 5 & 4 & 1 & 20 & V \\
\hline Mean & $42.8 /-$ & 21.8 & & & & 5.1 & 3.3 & 2.8 & 2.8 & 0.6 & 14.6 & \\
\hline
\end{tabular}

signed for contracture creating disfigurement. Summing the scores from each region, along with scores reflecting synkinesis, produced a final score ranging from 4 to 24 , which was converted to a grade ranging from I to VI as follows: grade I, FNGS score of 4; grade II, FNGS score of 5-9; grade III, FNGS score of 10-14; grade IV, FNGS score of 15-19; grade V, FNGS score of 20-23; and grade VI, FNGS of score 24 [1]. Functional outcomes were analyzed according to patient age, follow-up duration, and the use of postoperative radiation therapy.

\section{Statistical analysis}

The Wilcoxon rank-sum test was used to compare FNGS scores according to patient age ( $\leq 50$ years vs. $>50$ years), follow-up duration ( $\leq 18$ months vs. $>18$ months), and the use of postoperative radiation therapy (yes/no). A value of $\mathrm{P}<0.05$ was considered to indicate statistical significance.

\section{RESULTS}

All 12 patients in this series underwent reconstruction using fa- 
cial nerve cable grafts. The mean age at the time of surgery was 42.8 years, and the mean follow-up period was 21.8 months. The pathologic diagnoses were mucoepidermoid carcinoma $(n=4)$, squamous cell carcinoma $(\mathrm{n}=3)$, schwannoma $(\mathrm{n}=2)$, adenocarcinoma $(n=2)$, and sarcocarcinoma $(n=1)$. Seven of the 12 patients underwent postoperative radiation therapy. No woundrelated or microsurgical complications were noted (Table 1).

The average total FNGS score for the 12 patients was 14.6 (grade III), indicating moderate dysfunction. The average scores for individual facial regions were 5.1 (trace movement) for the forehead and brow area, 3.3 (good movement, $50 \%-75 \%$ of normal movement) for the periorbital area, and 2.8 (slight weakness, $>75 \%$ of normal movement) for both the nasolabial fold and perioral areas. These results demonstrate that better movement was observed in the lower face than in the upper face. Eight patients had lagophthalmos and could produce only slight wrinkles on their foreheads; however, they showed better movement of the muscles involved in facial expression at the nasolabial fold and oral commissure. The average score for synkinesis was 0.6, indicating slight or no synkinetic movement (Fig. 2).

Facial nerve function was analyzed according to age at the time of surgery, the use of radiotherapy, and follow-up duration. The mean FNGS score was 16.4 among patients older than 50 years, compared to 13.3 among those younger than 50 years; 14.3 among patients who underwent radiotherapy, compared to 15.0 among those who did not; and 15.2 among patients who underwent more than 18 months of follow-up, compared to 14.0 among those who underwent less than 18 months of follow-up. However, these differences were not significant (Fig. 3). Thus, our results reveal that patient age, the use of postoperative radiation therapy, and follow-up duration did not influence the FNGS score. Two of the 16 patients with parotid cancer experienced recurrence of the cancer, with a mean survival of 11.2 months.

\section{Case 1}

A 23-year-old female patient underwent radical parotidectomy for mucoepidermoid carcinoma of the right parotid gland (stage II) with concomitant facial nerve cable grafting using multiple

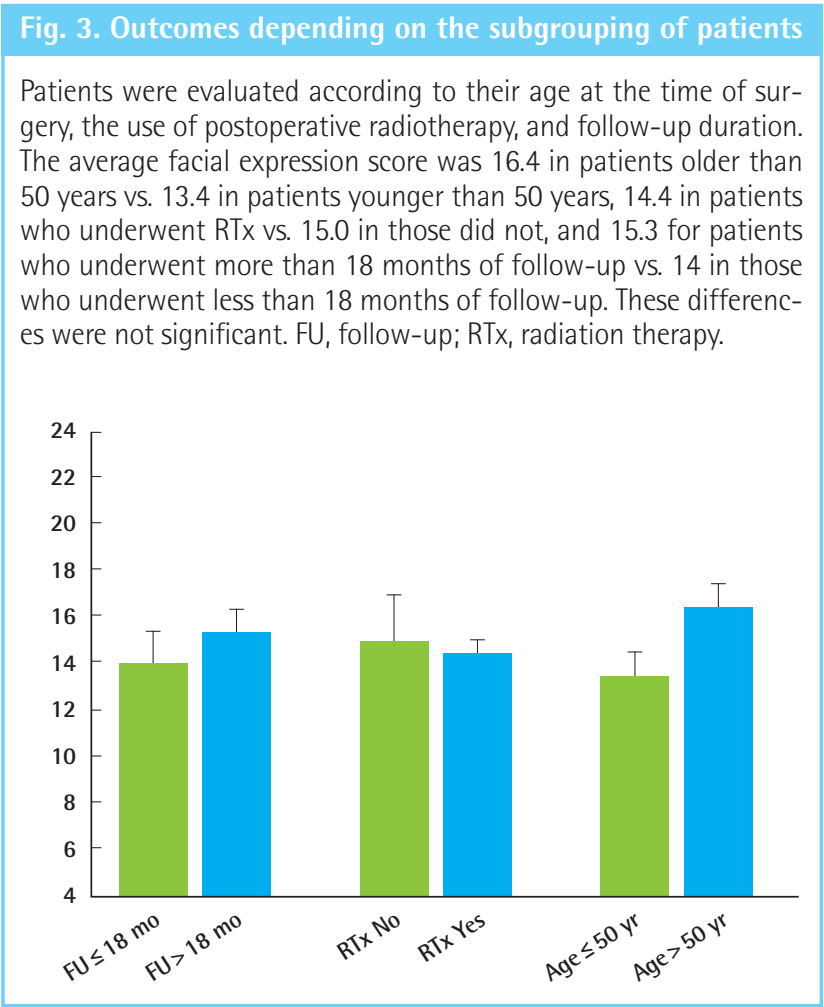

\section{Fig. 2. Average facial expression scores}

(A) The average overall score was 14.7 (grade III), indicating moderate dysfunction. (B) The regional scores were 5.1 for the forehead and brow area (trace movement), 3.4 for the periorbital area (good movement, 50\%-75\% of normal movement), and 2.8 for both the nasolabial fold (NLF) and perioral areas (slight weakness, $>75 \%$ of normal movement). The average synkinesis score was 0.58 .
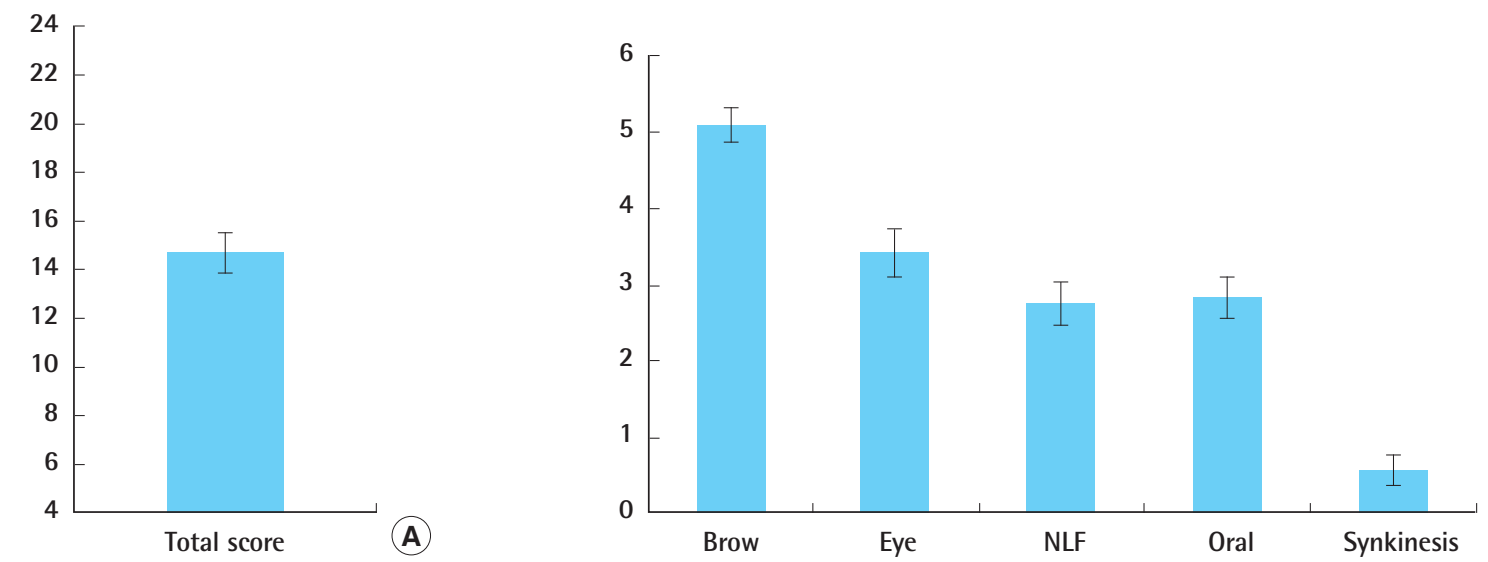
sural nerve segments. The patient underwent radiation therapy (5,750 cGy) postoperatively. Photos of her facial expressions taken nine months later showed that eye squeezing and movements at the nasolabial fold and oral commissure were fluent, but forehead wrinkling was limited, and a slight degree of synkinesis was present. Her regional FNGS score for forehead and brow movement (5) was worse than the scores for eye squeezing (2) and movements of the nasolabial fold (2) and oral commissure (2). The score for synkinesis was 1 , resulting in a total score of 12 (grade III) (Fig. 4).

\section{Case 2}

A 54-year-old female patient underwent total parotidectomy and supraomohyoid lymph node dissection for adenocarcinoma of the right parotid gland (stage III) with concomitant facial nerve cable grafting using multiple sural nerve segments. Photos of facial expressions taken 20 months later show eye-squeezing movements, although lagophthalmos was noted and forehead wrinkling was limited. The nasolabial fold and oral commissure showed symmetry at rest, but displayed obvious weakness while forming facial expressions. Her regional FNGS scores for brow and eye squeezing ( 4 and 4 , respectively) were worse than the

Fig. 4. Case I: assessment of facial expression

A 23-year-old female patient underwent radical parotidectomy for mucoepidermoid carcinoma of the right parotid gland (stage II), with concomitant facial nerve cable grafting; furthermore, she underwent radiation therapy $(5,750 \mathrm{cGy})$. Photos of (A) the resting state and $(B-E)$ facial expressions were taken nine months after surgery. The photos demonstrated that $(\mathrm{C})$ eye squeezing and movements of (D) the nasolabial fold and (E) the oral commissure were fluent; however (B) forehead wrinkling was limited, and a slight degree of synkinesis was present. The regional Facial Nerve Grading Scale 2.0 score for (B) forehead and brow movement (5) was worse than the scores for (C) eye squeezing (2) and the movements of (D) the nasolabial folds (2) and (E) the oral commissure (2). The synkinesis score was 1, resulting in a total score of 12 (grade III).

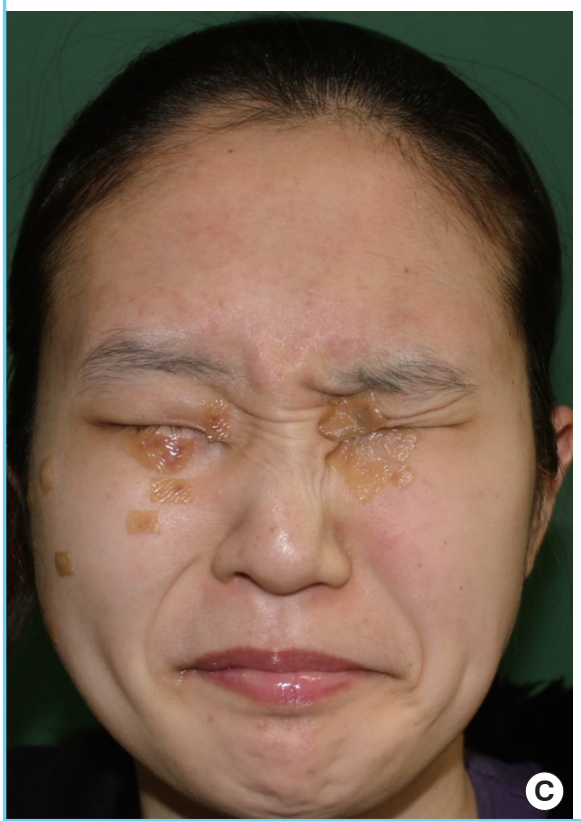

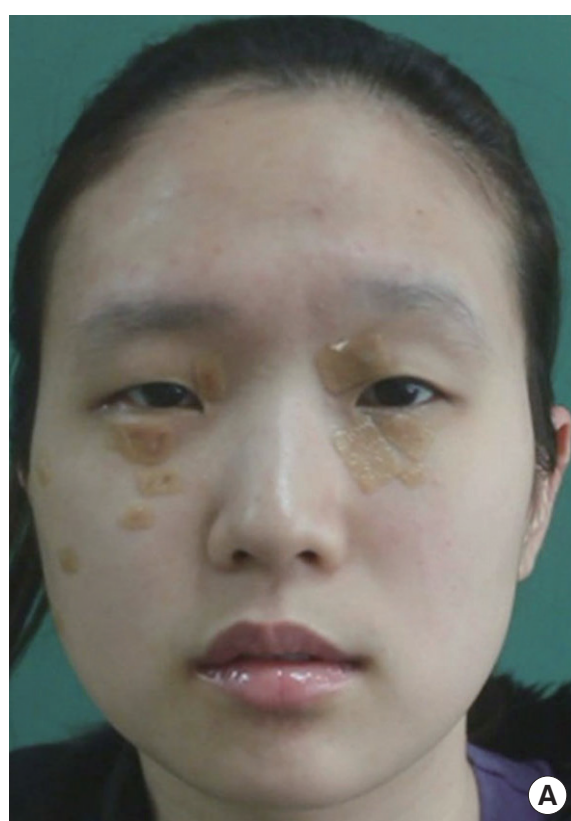
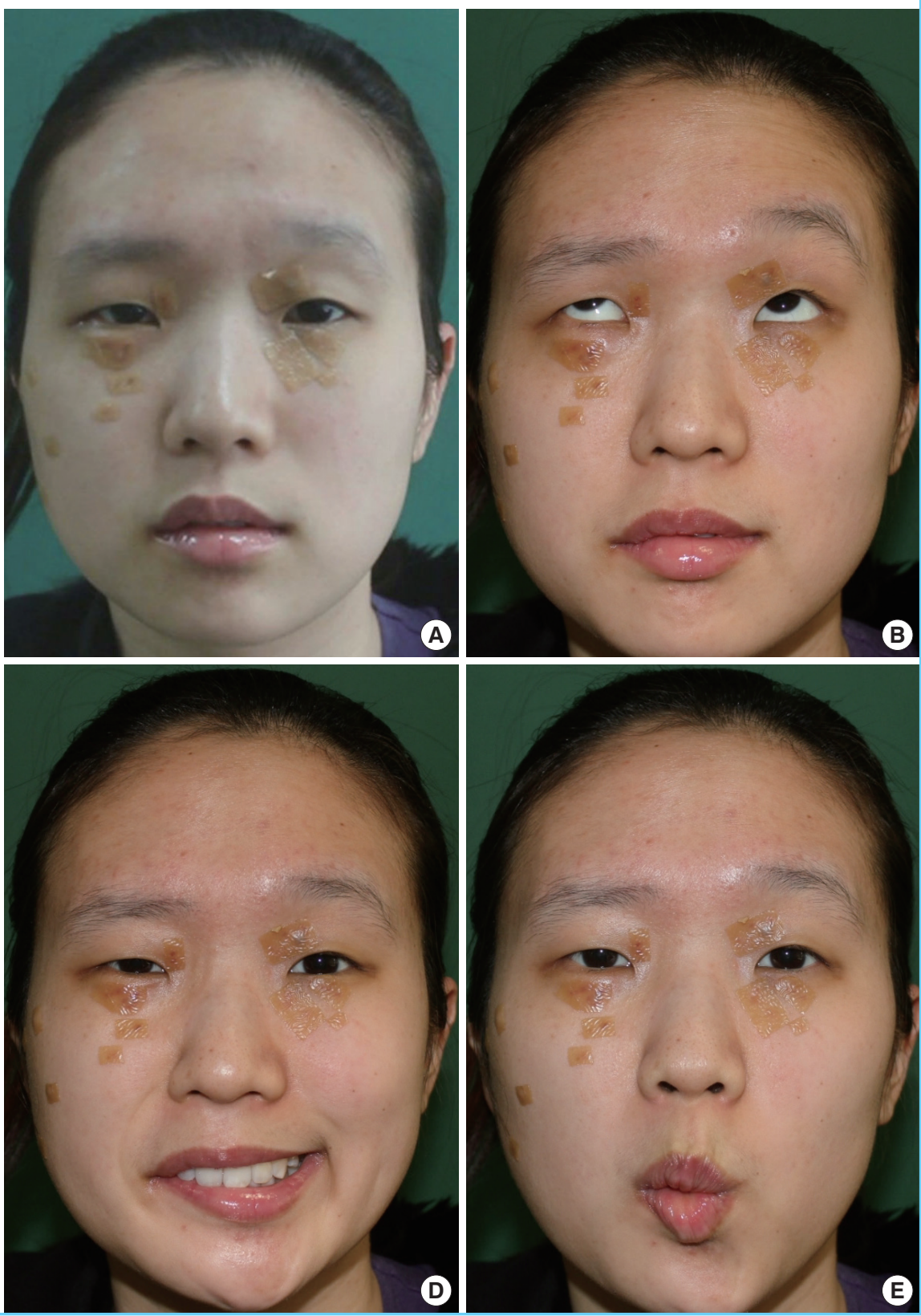


\section{Fig. 5. Case II: assessment of facial expression}

A 54-year-old female patient underwent total parotidectomy and supraomohyoid lymph node dissection for adenocarcinoma of the right parotid gland (stage III) with concomitant facial nerve cable grafting. Pictures of (A) the resting state and (B-E) facial expressions were taken 20 months after surgery. (C) The outcomes showed eye-squeezing movements, although lagophthalmos was noted. (B) Furthermore, forehead wrinkling was limited. (A) The nasolabial folds and oral commissure revealed resting symmetry, $(D, E)$ but obvious weakness was noted while forming facial expressions. The Regional Facial Nerve Grading Scale 2.0 scores for (B) the brow (4) and $(\mathrm{C})$ eye squeezing (4) were worse than the scores for (D) the nasolabial folds (3) and (E) the oral commissure (3). The synkinesis score was 1, leading to a total score of 15 (grade IV).
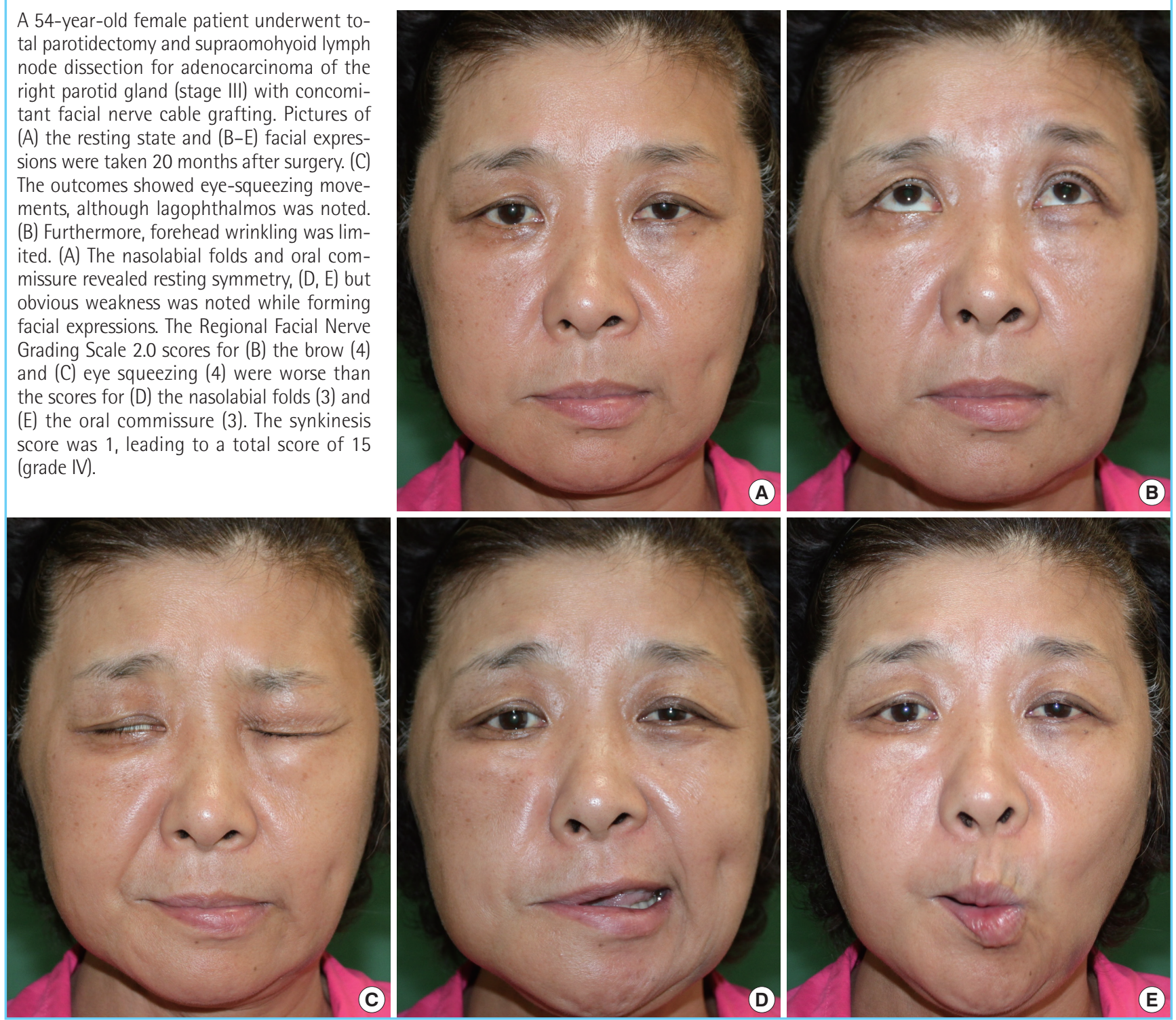

scores assessing the movement of the nasolabial folds (3) and oral commissure (3). The score for synkinesis was 1, resulting in a total score of 15 (grade IV) (Fig. 5).

\section{DISCUSSION}

Several surgical procedures have been introduced for the treatment of unilateral facial paralysis that occurs when the facial nerve is resected for tumor-ablative surgery. These procedures include direct facial nerve anastomosis, facial nerve cable grafts [6], anastomosis to other motor nerves, such as the hypoglossal nerve or spinal accessory nerve [1], dynamic muscular transposition using the temporalis or masseter muscle [13], static musculofascial slings, and combination procedures $[5]$. The facial nerve ca- ble grafting technique was first described by Conley [14] in 1955. He used the great auricular nerve to reconstruct facial nerve branches through cable grafting between the main trunk and five branches, and performed concomitant masseter muscle transposition after total parotidectomy. Several modifications have subsequently been introduced to improve his original technique.

Performing facial nerve cable grafting using the sural nerve is a well-established procedure for the repair of facial nerve defects. The sural nerve is sufficiently long and consists of multiple fascicles with interfascicular bridges. An anatomical study demonstrated the presence of 6.3 fascicles at the proximal end of the sural nerve and 6.7 fascicles at the distal end [10]. The facial nerve has a fascicular organization, with the perineurium and epineurium distal to the geniculate ganglion. The number of fascicles 
increases from the proximal to distal portion, and the diameter of the fascicles decreases toward the distal portion. Moreover, a previous study noted that an average of 13 fascicles were found in the temporofacial division and an average of 16 fascicles were found in the cervicofacial division, while the diameter of the nerve fibers varied from 0.1 to $0.8 \mathrm{~mm}$ [15]. The internal topography and multifascicular characteristics of the sural nerve allow successful anastomosis with the facial nerve, although discrepancies exist regarding the diameter of each nerve fiber.

The average FNGS score was grade III (average total score, 14.6) in our series of patients who underwent facial nerve cable grafting using the sural nerve. These results are equivalent to those of other surgical techniques that are currently used [6]. Recent studies of facial reanimation surgery after facial nerve damage have shown an average outcome of grade III or IV functionality (based on the House-Brackmann scale) [1-3]. In our results, movement of the lower face (nasolabial fold, oral commissure) was superior to that of the upper face (forehead, periorbital area). Almost all patients showed lagophthalmos and weakness in the forehead area; however, the function of the muscles involved in facial expression was better at the nasolabial fold and the oral commissure. This phenomenon may be due to the anatomy of the muscles involved in facial expression; the distance between the main trunk and the frontotemporal area is greater than the distance between the main trunk and the zygomatic area. Moreover, a greater quantity of coordinating muscles contribute to middle-lower face movement (zygomaticus major and minor, levator labii superioris, and risorius) than contribute to upper face and forehead movement (frontalis, orbicularis oculi). Synkinesis, which reflects the degree of nerve injury, has been classified by Sunderland [16] into five subgroups. When the endoneurium is disrupted, intrafascicular fibrosis may develop, which often blocks, delays, or diverts axonal growth during regeneration [6]. Axonal regeneration invariably results in misdirected fibers, leading to the clinical observation of synkinesis when the endoneurial sheath is disrupted in third through fifth degree injuries [17]. We observed little or no synkinetic movement in all of our patients, indicating that the sural nerve is an appropriate donor for facial nerve cable grafts and fits the topology of the facial nerve. These results could be attributed to the perineural repair of two strands of the sural nerve that became integrated into the upper and lower halves of the facial nerve main trunk, mimicking the temporofacial and cervicofacial divisions of the facial nerve. Moreover, the sural nerve branches were meticulously dissected, mimicking the arborization pattern of the facial nerve, and each fascicle was preserved and anastomosed to truncated peripheral facial nerve endings. This technique may have contributed to the favorable results that we observed.
FNGS scores did not differ significantly between patients older than 50 years and patients younger than 50 years. Similarly, patients who underwent radiotherapy did not have significantly different FNGS scores compared to those who did not, suggesting that surgeons should not hesitate to perform facial nerve grafting in patients who are older or who may require postoperative radiation therapy $[18,19]$. In our series, two of the 16 patients with parotid cancer experienced recurrence, with a mean survival of 11.2 months. The FNGS scores did not differ between the patients who underwent more than 18 months of follow-up and those who underwent less than 18 months of follow-up, suggesting that immediate facial nerve cable grafting is a useful procedure for restoring facial expression, thereby enhancing quality of life after facial nerve resection.

Hypoglossal-facial nerve transfer procedures, such as the hypoglossal-facial nerve jump graft [3] and a combination approach using a facial nerve interpositional graft with hypoglossal-facial jump nerve sutures [6], can achieve facial reanimation without synkinesis or hemitongue atrophy. However, these procedures are not optimal for restoring the smile, because they result in asynchronous expression [7]. Facial nerve cable grafting using the sural nerve achieves comparable results, without requiring a supplementary procedure or producing unwanted synkinetic movements.

Our results showed that this procedure can adequately restore facial expression. The cable grafting of two strands of the sural nerve to the facial nerve trunk, which was performed in order to replace the temporofacial and cervicofacial divisions of the facial nerve, contributed to overcoming the previously described problem of synkinesis. Although the patients received appropriate surgical intervention and concurrent chemoradiation therapy, the survival rate for salivary gland cancer was poor. Nonetheless, immediate facial nerve reconstruction with multiple cable grafts using the sural nerve is a worthwhile procedure for restoring facial expression and improving the quality of life, even in patients who are older or who may require postoperative radiation therapy.

\section{REFERENCES}

1. Catli T, Bayazit YA, Gokdogan O, et al. Facial reanimation with end-to-end hypoglossofacial anastomosis: 20 years' experience.J Laryngol Otol 2010;124:23-5.

2. Flores LP. Surgical results of the hypoglossal-facial nerve jump graft technique. Acta Neurochir (Wien) 2007;149:1205-10.

3. Reddy PG, Arden RL, Mathog RH. Facial nerve rehabilitation after radical parotidectomy. Laryngoscope 1999;109: 894-9.

4. Kakibuchi M, Tuji K, Fukuda K, et al. End-to-side nerve graft 
for facial nerve reconstruction. Ann Plast Surg 2004;53:496500.

5. Volk GF, Pantel M, Streppel M, et al. Reconstruction of complex peripheral facial nerve defects by a combined approach using facial nerve interpositional graft and hypoglossal-facial jump nerve suture. Laryngoscope 2011;121:2402-5.

6. Humphrey CD, Kriet JD. Nerve repair and cable grafting for facial paralysis. Facial Plast Surg 2008;24:170-6.

7. Chuang DC, Wei FC, Noordhoff MS. “Smile” reconstruction in facial paralysis. Ann Plast Surg 1989;23:56-65.

8. Wang Z, Zhang Z, Huang Q et al. Long-term facial nerve function following facial reanimation after translabyrinthine vestibular schwannoma surgery: a comparison between sural grafting and VII-XII anastomosis. Exp Ther Med 2013; 6:101-4.

9. Riedl O, Frey M. Anatomy of the sural nerve: cadaver study and literature review. Plast Reconstr Surg 2013;131:802-10.

10. Osinga R, Buncke HJ, Buncke GM, et al. Subdivision of the sural nerve: step towards individual facial reanimation. J Plast Surg Hand Surg 2011;45:3-7.

11. Myckatyn TM, Mackinnon SE. A review of facial nerve anatomy. Semin Plast Surg 2004;18:5-12.

12. Vrabec JT, Backous DD, Djalilian HR, et al. Facial Nerve Grading System 2.0. Otolaryngol Head Neck Surg 2009;140:
445-50.

13. Rubin LR. Re-animation of total unilateral facial paralysis by the contiguous facial muscle technique. In: Rubin LR, editor. The paralyzed face. St. Louis: Mosby Year Book; 1991. p.156-77.

14. Conley JJ. Facial nerve grafting in treatment of parotid gland tumors; new technique. AMA Arch Surg 1955;70:359-66.

15. Captier G, Canovas F, Bonnel F, et al. Organization and microscopic anatomy of the adult human facial nerve: anatomical and histological basis for surgery. Plast Reconstr Surg 2005; 115:1457-65.

16. Sunderland S. The anatomy and physiology of nerve injury. Muscle Nerve 1990;13:771-84.

17. Filipo R, Spahiu I, Covelli E, et al. Botulinum toxin in the treatment of facial synkinesis and hyperkinesis. Laryngoscope 2012;122:266-70.

18. Brown PD, Eshleman JS, Foote RL, et al. An analysis of facial nerve function in irradiated and unirradiated facial nerve grafts. Int J Radiat Oncol Biol Phys 2000;48:737-43.

19. Hontanilla B, Qiu SS, Marre D. Effect of postoperative brachytherapy and external beam radiotherapy on functional outcomes of immediate facial nerve repair after radical parotidectomy. Head Neck 2014;36:113-9. 